\title{
Comparison of carbetocin and oxytocin infusions in reducing the requirement for additional uterotonics or procedures in women at increased risk of postpartum haemorrhage after Caesarean section
}

\author{
KY Tse *, Florrie NY Yu, KY Leung
}

\section{A B S T R A C T}

Introduction: Postpartum haemorrhage is a major cause of maternal mortality and morbidity, commonly due to uterine atony. Prophylactic oxytocin use during Caesarean section is recommended; patients with a high risk of postpartum haemorrhage may require additional uterotonics or procedures. Carbetocin is a long-acting analogue of oxytocin which has shown beneficial results, compared with oxytocin. This study compared the requirement for additional uterotonics or procedures between at-risk women who underwent carbetocin infusion and those who underwent oxytocin infusion.

Methods: This retrospective cohort study included women at increased risk of postpartum haemorrhage after Caesarean section for various indications in a public hospital. Women who received carbetocin infusion and women who received oxytocin infusion were compared, stratified by Caesarean section timing (elective or emergency). The primary outcome was the requirement for additional uterotonic agents or procedures. Secondary outcomes included total blood loss, operating time, rate of postpartum haemorrhage, need for blood transfusion, and need
This article was published on 8 Oct 2020 at www.hkmj.org.
Results: Of 1236 women included in the study, 752 received oxytocin first and 484 received carbetocin first. The two groups had comparable blood loss, operating time, rate of postpartum haemorrhage, requirement for additional uterotonics or procedures, need for blood transfusion, and need for hysterectomy. There was a reduction in the requirement for additional uterotonics or procedures, and in the rate of postpartum haemorrhage for women with major placenta praevia or with multiple pregnancies, following receipt of carbetocin first.

Conclusion: Compared with oxytocin, carbetocin can reduce the requirement for additional uterotonics or procedures in selected high-risk patient groups.
Hong Kong Med J 2020;26:382-9
https://doi.org/10.12809/hkmj208683
KY Tse *, MB, BS, MRCOG
FNY Yu, FHKAM (Obstetrics and Gynaecology)
KY Leung, FRCOG
Department of Obstetrics and Gynaecology, Queen Elizabeth Hospital, Hong Kong
* Corresponding author: barontse@hotmail.com

New knowledge added by this study

- The use of carbetocin reduced the requirement for additional uterotonics or procedures in women with major placenta praevia and in women with multiple pregnancies.

- Infusions of carbetocin and oxytocin had differential effects on the requirement for additional uterotonics or procedures in women who underwent Caesarean section for different indications.

- Women who received carbetocin infusion had similar blood loss, operating time, rate of postpartum haemorrhage, requirement for additional uterotonics or procedures, need for blood transfusion, and need for hysterectomy, compared with women who received oxytocin infusion.

Implications for clinical practice or policy

- Carbetocin may be appropriate for women undergoing Caesarean section for major placenta praevia or multiple pregnancies.

- Oxytocin may be appropriate for women undergoing Caesarean section for other indications.

- There is a need to investigate the cost-effectiveness of carbetocin, which will aid clinicians in treatment selection.

\section{Introduction}

This type of haemorrhage is defined as blood loss of at

Postpartum haemorrhage is the major cause of maternal death and morbidity worldwide, ${ }^{1}$ commonly due to uterine atony (approximately $70 \%$ of cases). ${ }^{2}$ least $500 \mathrm{~mL}$ after vaginal delivery and blood loss of $>1000 \mathrm{~mL}$ after Caesarean section. ${ }^{3}$ Oxytocin (with or without ergometrine) is the current standard therapy 
for the prevention of postpartum haemorrhage; it is a peptide hormone secreted by the posterior pituitary gland, which stimulates myometrial contraction in the second and third stages of labour. However, failure of postpartum haemorrhage prophylaxis with oxytocin (as demonstrated by the need for a rescue uterotonic) occurs commonly, necessitating the use of further oxytocin or other treatments to maintain haemodynamic stability. ${ }^{4}$

Carbetocin is a synthetic analogue of oxytocin which has a longer half-life than oxytocin, thus reducing the requirement for an infusion after the initial dose. This difference in structure, compared with oxytocin, makes carbetocin more stable; thus, carbetocin can avoid early decomposition by disulfidase, aminopeptidase, and oxidoreductase enzymes. Compared with oxytocin, carbetocin induces a prolonged uterine response, in terms of both amplitude and frequency of contractions, when administered postpartum. ${ }^{5}$

A Cochrane review in 2012 found that carbetocin reduced the use of additional uterotonics and uterine massage, when compared with oxytocin. $^{3}$ In 2018, a meta-analysis involving seven trials showed that carbetocin was effective in reducing the use of additional uterotonics, as well as in reducing postpartum haemorrhage and transfusion, when used during Caesarean section. ${ }^{6}$ A recent meta-analysis involving 30 trials has shown that carbetocin is effective in reducing the need for additional uterotonic use and postpartum blood transfusion in women at increased risk of postpartum haemorrhage after Caesarean delivery. ${ }^{7}$ Another recent meta-analysis involving nine trials has shown that carbetocin is associated with a 53\% reduction in the need for additional uterotonics, when compared with oxytocin, at the time of elective Caesarean delivery. ${ }^{8}$ The use of carbetocin has been recommended in elective Caesarean sections by the Royal College of Obstetricians and Gynaecologists ${ }^{9}$ and the Society of Obstetricians and Gynaecologists of Canada. ${ }^{10}$

In one meta-analysis, ${ }^{6}$ heterogeneity was found in the use of dose of oxytocin, which ranged from 2 IU to $10 \mathrm{IU}$ of bolus oxytocin to infusions of 20 to $30 \mathrm{IU}$, and in the indications for Caesarean section. In a study of 1568 Chinese women who underwent Caesarean section for different indications, carbetocin and oxytocin were found to have differential effects on postpartum haemorrhage and related changes. ${ }^{6}$ However, to the best of our knowledge, most studies have compared the differential therapeutic effects of carbetocin and oxytocin in the general population or in low-risk groups. Carbetocin is a relatively more expensive drug than conventional synthetic oxytocin (eg, Syntocinon), and there has not been sufficient evidence from cost-effectiveness studies to support its use in all patients. The use of carbetocin is therefore

\section{剖腹產後出血風險增加的婦女，使用卡貝縮宮素 和催產素輸注減少對其他子宮內縮劑或手術需求 的比較}

\author{
謝啓暘、俞雅芯、梁國賢
}

引言：產後出血是產婦死亡和發病的主因, 通常是子宮收縮乏力所 致。建議在剖腹產時使用預防性催產素。產後出血風險高的患者可能 需要額外的子宮收縮劑或其他手術。卡貝縮宮素是催產素的長效類似 物, 與催產素相比顯示有益成果。本研究將卡貝縮宮素輸注和催產素 輸注的高危婦女對額外子宮內縮劑治療或手術需求作比較

方法：這項回顧性隊列研究納入於公立醫院剖腹產後因各種適應症增 加出血風險的婦女。將卡貝縮宮素輸注和催產素輸注的婦女作比較, 並按剖腹產時機（選擇性或緊急）進行分層。主要結果包括額外子宮 收縮劑或手術的需求。次要結果包括總失血量、手術時間、產後出血 率、對輸血和子宮切除術的需求。

結果: 納入研究的 1236 名婦女中, 752名先接受催產素, 484名先接 受卡貝縮宮素。兩組的失血量、手術時間、產後出血率、對額外子宮 收縮劑或手術的需求、輸血和子宮切除需要的情況相若。接受卡貝縮 宮素後, 患有重度前置胎盤或多胎妊娠的婦女對額外子宮收縮劑或手 術以及產後出血的比率皆有所下降。

結論：與催產素相比，卡貝縮宮素可減少高危患者對額外子宮收縮齊 或手術的需求。

limited to certain high-risk populations in some institutions, including our hospital. The aim of the present study was to compare effectiveness between carbetocin and oxytocin in terms of reducing the requirement for additional uterotonics in women at increased risk of postpartum haemorrhage after Caesarean section.

\section{Methods}

This study was conducted at the Department of Obstetrics and Gynaecology, Queen Elizabeth Hospital, Hong Kong, from 1 January 2016 to 30 June 2019; during this period, 5994 pregnant women underwent Caesarean section in our hospital. Demographic characteristics, obstetric histories, risk factors, and perinatal outcomes were collected. The study was approved by the Hospital Authority Research Ethics Committee (Kowloon Central/ Kowloon East) [KC/KE-20-0073/ER-1].

The inclusion criteria were women who underwent Caesarean section for live birth after 24 weeks of completed gestation during the aforementioned time period, with high risk of developing postpartum haemorrhage (including placenta praevia, presence of uterine fibroids, multiple pregnancies, polyhydramnios, and macrosomia), excluding patients with low risk ( $n=4758$, of whom 96 had low risk but received oxytocin and carbetocin after identification of postpartum haemorrhage). Patients who received both oxytocin and carbetocin were assigned to the 
group where the first drug (oxytocin or carbetocin) was used for prevention of postpartum haemorrhage, then considered to require additional uterotonics (carbetocin or oxytocin).

In our hospital, the implementation of carbetocin began in April 2017. Prior to the implementation of carbetocin, women who were presumed to have a low risk of postpartum haemorrhage were administered 10 IU oxytocin intravenous bolus after delivery of the baby during Caesarean section; women who were presumed to have a high risk of postpartum haemorrhage were administered 40 IU oxytocin intravenous infusion over the course of 5 hours for a longer protective effect. Depending on the clinical response, further infusion of oxytocin might be indicated. Following the implementation of carbetocin, we have revised our protocol to administer the drug $100 \mu \mathrm{g}$ intravenously over 1 minute for a single dose after delivery of the baby, for women with a high risk of postpartum haemorrhage (ie, with the aforementioned risk factors) or for women who required such treatment in accordance with the obstetrician's judgement. Contra-indications included hypersensitivity to carbetocin, timing prior to delivery of the baby, vascular disease (especially coronary artery disease), and hepatic or renal disease. The use of blood transfusion, additional uterotonic agents (eg, carboprost $250 \mu \mathrm{g}$ intramuscularly or intramyometrially, misoprostol $800 \mu \mathrm{g}$ rectally, oxytocin infusion after carbetocin, or carbetocin after oxytocin infusion), obstetric balloon tamponade, compression suture, uterine artery embolisation, uterine artery ligation, or hysterectomy was based on the control of postpartum haemorrhage and the patient's vital signs, as well as the attending obstetrician's judgement.

In this study, we subdivided the patients according to risk factors. We then analysed each patient group separately: patients who underwent elective Caesarean section (ie, those who underwent Caesarean section before labour onset) versus patients who underwent emergency Caesarean section (ie, those who either underwent intrapartum Caesarean section, or who underwent Caesarean section prior to the scheduled elective date for reasons such as heavy antepartum haemorrhage).

The primary outcome was the requirement for additional uterotonic agents or haemostatic procedures (carboprost, misoprostol, oxytocin infusion after carbetocin, carbetocin after oxytocin infusion, obstetric balloon tamponade, uterine artery embolisation, or uterine artery ligation). Secondary outcomes were estimated blood loss, rate of postpartum haemorrhage, operating time, the need for blood transfusion, and the need for hysterectomy. Blood loss was estimated by measuring the volume within the suction bottle and the uptake in surgical drapes, pads, and gauzes. Postpartum haemorrhage was defined as blood loss $>1000 \mathrm{~mL}$ during or immediately after the operation.

Statistical analysis was performed using IBM SPSS Statistics for Windows, version 20.0 (IBM Corp, Armonk [NY], United States). Continuous variables were expressed as the mean \pm standard deviation and compared by Student's $t$ test. Qualitative data were expressed as number (percentage) and compared by the Chi squared test or Fisher's exact test. A P value of $<0.05$ was considered statistically significant. Linear regression and multiple logistic regression were used to control for potential confounding factors when assessing the associations of carbetocin treatment with primary outcomes. Potential confounding factors included age, parity, fetal body weight, gestation, and order of pregnancy. Unstandardised regression coefficients, adjusted odds ratios, and associated $95 \%$ confidence intervals were calculated to estimate relative risk.

\section{Results}

Of 5994 pregnant women who underwent Caesarean sections during the study period, 4758 were excluded because they were at low risk of postpartum haemorrhage. Of the remaining 1236 women who met the criteria for inclusion in the study, 752 received oxytocin first and 484 received carbetocin first. Compared with women who received oxytocin first, women who received carbetocin first had earlier gestation, lower neonatal birth weight, and a greater proportion of twins or higher order pregnancy (Table 1). The two groups had comparable blood loss, operating time, rate of postpartum haemorrhage, requirement for additional uterotonics and procedures, need for blood transfusion, and need for hysterectomy (Table 2).

Significant reductions in the rate of postpartum haemorrhage and in the requirement for additional uterotonics or procedures were observed among women with multiple pregnancies (Table 3a) and women with major placenta praevia (Table $3 \mathrm{~b}$ ). Significant reductions in total blood loss were also observed for women with multiple pregnancies and women with major placenta praevia in the emergency Caesarean group. Additionally, the rate of hysterectomy was significantly reduced in women with multiple pregnancies in the emergency Caesarean group.

For women with minor placenta praevia (Table 3c), fibroids (Table 3d), macrosomia (Table 3e), or polyhydramnios (Table 3f), no significant differences in total blood loss, operating time, rate of postpartum haemorrhage, requirement for additional uterotonics, need for blood transfusion, or need for hysterectomy were observed between the two groups. 
TABLE I. Maternal demographics of the study population*

\begin{tabular}{|c|c|c|c|c|c|c|c|c|c|}
\hline & \multicolumn{3}{|c|}{ Elective Caesarean section } & \multicolumn{3}{|c|}{ Emergency Caesarean section } & \multicolumn{3}{|c|}{ Total } \\
\hline & $\begin{array}{l}\text { Oxytocin } \\
(n=347)\end{array}$ & $\begin{array}{l}\text { Carbetocin } \\
(n=202)\end{array}$ & $P$ value & $\begin{array}{l}\text { Oxytocin } \\
(n=405)\end{array}$ & $\begin{array}{l}\text { Carbetocin } \\
(n=282)\end{array}$ & $P$ value & $\begin{array}{c}\text { Oxytocin } \\
(n=752)\end{array}$ & $\begin{array}{c}\text { Carbetocin } \\
(n=484)\end{array}$ & $P$ value \\
\hline Age (years) $\dagger$ & $33.9 \pm 4.5$ & $34.5 \pm 5.0$ & 0.17 & $34.2 \pm 4.2$ & $34.3 \pm 4.4$ & 0.70 & $34.1 \pm 4.3$ & $34.4 \pm 4.7$ & 0.20 \\
\hline Gestation (weeks)† & $37.0 \pm 2.4$ & $36.2 \pm 2.5$ & $<0.01$ & $37.1 \pm 2.3$ & $35.8 \pm 2.8$ & $<0.01$ & $37.1 \pm 2.3$ & $36.0 \pm 2.7$ & $<0.01$ \\
\hline Neonatal birth weight $(\mathrm{g}) \dagger$ & $2887.4 \pm 766.0$ & $2612.1 \pm 719.2$ & $<0.01$ & $2962.1 \pm 793.0$ & $2505.9 \pm 737.7$ & $<0.01$ & $2927.7 \pm 781.1$ & $2549.0 \pm 729.7$ & $<0.01$ \\
\hline Parity† & $0.5 \pm 0.6$ & $0.5 \pm 0.8$ & 0.41 & $0.5 \pm 0.7$ & $0.4 \pm 0.7$ & 0.16 & $0.5 \pm 0.7$ & $0.5 \pm 0.7$ & 0.57 \\
\hline \multicolumn{10}{|l|}{ No. of fetuses $\ddagger$} \\
\hline Singleton & $230(66.3 \%)$ & $106(52.5 \%)$ & & $281(69.4 \%)$ & $148(52.5 \%)$ & & $511(68.0 \%)$ & $254(52.5 \%)$ & \\
\hline Multiple pregnancy & $117(33.7 \%)$ & $96(47.5 \%)$ & 0.01 & $124(30.6 \%)$ & 134 (47.5\%) & $<0.01$ & 241 (32.0\%) & 230 (47.5\%) & $<0.01$ \\
\hline
\end{tabular}

* Data are shown as No. (\%) or mean \pm standard deviation, unless otherwise specified

† Data calculated using Student's $t$ test

‡ Data calculated using Chi squared test

TABLE 2. Comparisons of the effects of oxytocin and carbetocin treatments on obstetric outcomes in all patients with high risk of postpartum haemorrhage*

\begin{tabular}{|c|c|c|c|c|c|c|c|c|c|}
\hline & \multicolumn{3}{|c|}{ Elective Caesarean section } & \multicolumn{3}{|c|}{ Emergency Caesarean section } & \multicolumn{3}{|c|}{ Total } \\
\hline & $\begin{array}{l}\text { Oxytocin } \\
(n=347)\end{array}$ & $\begin{array}{l}\text { Carbetocin } \\
(n=202)\end{array}$ & $P$ value & $\begin{array}{c}\text { Oxytocin } \\
(n=405)\end{array}$ & $\begin{array}{l}\text { Carbetocin } \\
(\mathbf{n}=282)\end{array}$ & $P$ value & $\begin{array}{l}\text { Oxytocin } \\
(n=752)\end{array}$ & $\begin{array}{c}\text { Carbetocin } \\
(n=484)\end{array}$ & $P$ value \\
\hline Total blood loss $(\mathrm{mL}) \dagger$ & $510.1 \pm 471.4$ & $572.6 \pm 761.5$ & 0.30 & $568.7 \pm 899.1$ & $514.0 \pm 356.9$ & 0.33 & $540.9 \pm 732.5$ & $536 \pm 559.5$ & 0.91 \\
\hline Operating time (minutes) $\dagger$ & $40.8 \pm 18.1$ & $44.6 \pm 28.4$ & 0.06 & $42.0 \pm 24.0$ & $41.0 \pm 14.4$ & 0.50 & $41.5 \pm 21.5$ & $42.5 \pm 21.3$ & 0.44 \\
\hline $\begin{array}{l}\text { Postpartum } \\
\text { haemorrhage }\end{array}$ & $32(9.2 \%)$ & $15(7.4 \%)$ & 0.46 & $29(7.2 \%)$ & $23(8.2 \%)$ & 0.63 & $61(8.1 \%)$ & 38 (7.9\%) & 0.87 \\
\hline $\begin{array}{l}\text { Additional uterotonic } \\
\text { agents or procedures } \ddagger\end{array}$ & $23(6.6 \%)$ & $11(5.4 \%)$ & 0.41 & $25(6.2 \%)$ & $15(5.3 \%)$ & 0.79 & $48(6.4 \%)$ & $26(5.4 \%)$ & 0.47 \\
\hline Blood transfusion $\ddagger$ & $22(6.3 \%)$ & $18(8.9 \%)$ & 0.27 & $18(4.4 \%)$ & $27(9.6 \%)$ & 0.06 & $40(5.3 \%)$ & $45(9.3 \%)$ & 0.07 \\
\hline Hysterectomy§ & $1(0.3 \%)$ & $2(1.0 \%)$ & 0.30 & $6(1.5 \%)$ & $1(0.4 \%)$ & 0.25 & $7(0.9 \%)$ & $3(0.6 \%)$ & 0.75 \\
\hline
\end{tabular}

* Data are shown as No. (\%) or mean \pm standard deviation, unless otherwise specified

† Data calculated using Student's $t$ test

‡ Data calculated using Chi squared test

$\S$ Data calculated using Fisher's exact test

After adjustments for confounding effects, linear regression analysis revealed reductions in the rate of postpartum haemorrhage and in the requirement for additional uterotonics or procedures for Caesarean section in women with major placenta praevia and in women with multiple pregnancies, but not for other risk factors (Table 4).

No serious side-effects were reported after the use of carbetocin.

\section{Discussion}

This study showed that the general cohort of women with risk factors for postpartum haemorrhage who received carbetocin treatment exhibited comparable blood loss, rate of postpartum haemorrhage, requirement for additional uterotonics or procedures, need for blood transfusion, and need for hysterectomy, compared with oxytocin treatment.
Our results are inconsistent with the findings of previous studies, ${ }^{11-14}$ in which there were reductions in blood loss and risk of postpartum haemorrhage among women in the general population following receipt of carbetocin alone, compared with oxytocin alone. We presume that the difference was related partly to the use of different oxytocin regimens. In particular, 40 IU oxytocin infusion (greater than the effective dose of carbetocin, 10 IU oxytocin) was used in the present study, whereas a smaller dose of oxytocin infusion of 10 to $20 \mathrm{IU}$ or a bolus of $5 \mathrm{IU}$ was used in previous studies. ${ }^{4,15,16}$ Furthermore, the indications for Caesarean section might have differed between the present study and the prior studies. Notably, carbetocin and oxytocin have been shown to exert differential effects on postpartum haemorrhage and related changes in patients undergoing Caesarean section for different indications. ${ }^{6}$ 
TABLE 3. Comparisons of the effects of oxytocin and carbetocin treatment on obstetric outcomes in patients with various risk factors (a) Twins or triplets*

\begin{tabular}{|c|c|c|c|c|c|c|c|c|c|}
\hline & \multicolumn{3}{|c|}{ Elective Caesarean section } & \multicolumn{3}{|c|}{ Emergency Caesarean section } & \multicolumn{3}{|c|}{ Total } \\
\hline & $\begin{array}{l}\text { Oxytocin } \\
(n=113)\end{array}$ & $\begin{array}{c}\text { Carbetocin } \\
(n=93)\end{array}$ & $P$ value & $\begin{array}{l}\text { Oxytocin } \\
(n=128)\end{array}$ & $\begin{array}{l}\text { Carbetocin } \\
(n=137)\end{array}$ & $P$ value & $\begin{array}{l}\text { Oxytocin } \\
(\mathrm{n}=241)\end{array}$ & $\begin{array}{l}\text { Carbetocin } \\
(n=230)\end{array}$ & $P$ value \\
\hline Total blood loss $(\mathrm{mL}) \dagger$ & $509.6 \pm 337.4$ & $540.3 \pm 640.2$ & 0.68 & $794.4 \pm 141.6$ & $490.7 \pm 337.0$ & 0.02 & $657.2 \pm 1071.3$ & $509.1 \pm 482.5$ & 0.06 \\
\hline Operating time $(\min ) \dagger$ & $39.8 \pm 19.6$ & $44.6 \pm 30.7$ & 0.20 & $43.7 \pm 35.3$ & $38.9 \pm 11.2$ & 0.14 & $41.7 \pm 28.8$ & $41.1 \pm 21.5$ & 0.26 \\
\hline Postpartum haemorrhage $\ddagger$ & $14(12.4 \%)$ & $6(6.5 \%)$ & 0.15 & $14(10.9 \%)$ & $8(5.8 \%)$ & 0.13 & $28(11.6 \%)$ & $14(6.1 \%)$ & 0.04 \\
\hline $\begin{array}{l}\text { Additional uterotonic } \\
\text { agents or proceduresł }\end{array}$ & $10(8.8 \%)$ & $4(4.3 \%)$ & 0.20 & $12(9.4 \%)$ & $5(3.6 \%)$ & 0.06 & $22(9.1 \%)$ & $9(3.9 \%)$ & 0.02 \\
\hline Blood transfusion $\ddagger$ & $9(8.0 \%)$ & $9(9.7 \%)$ & 0.66 & $9(7.0 \%)$ & $14(10.2 \%)$ & 0.25 & $18(7.5 \%)$ & $23(10.0 \%)$ & 0.33 \\
\hline Hysterectomy§ & 0 & 0 & - & $5(3.9 \%)$ & 0 & 0.03 & $5(2.1 \%)$ & 0 & 0.06 \\
\hline
\end{tabular}

(b) Placenta praevia (major type)*

\begin{tabular}{|c|c|c|c|c|c|c|c|c|c|}
\hline & \multicolumn{3}{|c|}{ Elective Caesarean section } & \multicolumn{3}{|c|}{ Emergency Caesarean section } & \multicolumn{3}{|c|}{ Total } \\
\hline & $\begin{array}{c}\text { Oxytocin } \\
(n=14)\end{array}$ & $\begin{array}{l}\text { Carbetocin } \\
(n=24)\end{array}$ & $\begin{array}{c}P \\
\text { value }\end{array}$ & $\begin{array}{l}\text { Oxytocin } \\
(n=21)\end{array}$ & $\begin{array}{c}\text { Carbetocin } \\
(n=32)\end{array}$ & $\begin{array}{c}P \\
\text { value }\end{array}$ & $\begin{array}{l}\text { Oxytocin } \\
(n=35)\end{array}$ & $\begin{array}{c}\text { Carbetocin } \\
(n=56)\end{array}$ & $\begin{array}{c}P \\
\text { value }\end{array}$ \\
\hline Total blood loss $(\mathrm{mL}) \dagger$ & $1132.1 \pm 1727.9$ & $1120.8 \pm 1639.3$ & 0.98 & $1285.7 \pm 1675.5$ & $656.3 \pm 463.5$ & 0.05 & $1224.3 \pm 1672.9$ & $855.4 \pm 1140.0$ & 0.26 \\
\hline Operating time (minutes) $\dagger$ & $51.6 \pm 34.0$ & $60.5 \pm 47.2$ & 0.51 & $56.3 \pm 43.4$ & $45.4 \pm 20.2$ & 0.29 & $54.4 \pm 39.4$ & $51.8 \pm 35.0$ & 0.76 \\
\hline Postpartum haemorrhage $\neq$ & $4(28.6 \%)$ & $4(16.7 \%)$ & 0.39 & $8(38.1 \%)$ & $4(12.5 \%)$ & 0.03 & $12(34.3 \%)$ & $8(14.3 \%)$ & 0.03 \\
\hline $\begin{array}{l}\text { Additional uterotonic } \\
\text { agents or procedures } \ddagger\end{array}$ & $3(21.4 \%)$ & $4(16.7 \%)$ & 0.72 & $7(33.3 \%)$ & $2(6.3 \%)$ & 0.01 & $10(28.6 \%)$ & $6(10.7 \%)$ & 0.03 \\
\hline Blood transfusion $\ddagger$ & $3(21.4 \%)$ & $6(25.0 \%)$ & 0.80 & $5(23.8 \%)$ & $8(25.0 \%)$ & 0.92 & $8(22.9 \%)$ & $14(25.0 \%)$ & 0.82 \\
\hline Hysterectomy§ & 1 (7.1\%) & 2 (8.3\%) & 1.00 & $1(4.8 \%)$ & $1(3.1 \%)$ & 1.00 & $2(5.7 \%)$ & 3 (5.4\%) & 1.00 \\
\hline
\end{tabular}

(c) Placenta praevia (minor type)*

\begin{tabular}{|c|c|c|c|c|c|c|c|c|c|}
\hline & \multicolumn{3}{|c|}{ Elective Caesarean section } & \multicolumn{3}{|c|}{ Emergency Caesarean section } & \multicolumn{3}{|c|}{ Total } \\
\hline & $\begin{array}{l}\text { Oxytocin } \\
(n=19)\end{array}$ & $\begin{array}{l}\text { Carbetocin } \\
(n=35)\end{array}$ & $P$ value & $\begin{array}{l}\text { Oxytocin } \\
(n=22)\end{array}$ & $\begin{array}{l}\text { Carbetocin } \\
(n=64)\end{array}$ & $P$ value & $\begin{array}{l}\text { Oxytocin } \\
(n=41)\end{array}$ & $\begin{array}{l}\text { Carbetocin } \\
(n=99)\end{array}$ & $P$ value \\
\hline Total blood loss $(\mathrm{mL}) \dagger$ & $676.3 \pm 504.5$ & $534.3 \pm 323.8$ & 0.28 & $518.2 \pm 239.8$ & $504.7 \pm 363.6$ & 0.84 & $591.5 \pm 388.7$ & $515.1 \pm 348.6$ & 0.17 \\
\hline Operating time (minutes) $\dagger$ & $38.3 \pm 15.1$ & $39.3 \pm 9.8$ & 0.79 & $36.0 \pm 13.7$ & $41.4 \pm 14.6$ & 0.12 & $37.1 \pm 14.2$ & $40.7 \pm 13.1$ & 0.28 \\
\hline Postpartum haemorrhage $\ddagger$ & $3(15.8 \%)$ & $3(8.6 \%)$ & 0.42 & $1(4.5 \%)$ & $6(9.4 \%)$ & 0.48 & $4(9.8 \%)$ & 9 (9.1\%) & 0.90 \\
\hline $\begin{array}{l}\text { Additional uterotonic } \\
\text { agents or proceduresł }\end{array}$ & $3(15.8 \%)$ & $2(5.7 \%)$ & 0.22 & 0 & $3(4.7 \%)$ & 0.30 & $3(7.3 \%)$ & $5(5.1 \%)$ & 0.60 \\
\hline Blood transfusion $\ddagger$ & $3(15.8 \%)$ & $4(11.4 \%)$ & 0.65 & 0 & $3(4.7 \%)$ & 0.30 & $3(7.3 \%)$ & $7(7.1 \%)$ & 0.96 \\
\hline Hysterectomy§ & 0 & 0 & - & 0 & 0 & - & 0 & 0 & - \\
\hline
\end{tabular}

(d) Fibroids*

\begin{tabular}{|c|c|c|c|c|c|c|c|c|c|}
\hline & \multicolumn{3}{|c|}{ Elective Caesarean section } & \multicolumn{3}{|c|}{ Emergency Caesarean section } & \multicolumn{3}{|c|}{ Total } \\
\hline & $\begin{array}{c}\text { Oxytocin } \\
(n=108)\end{array}$ & $\begin{array}{l}\text { Carbetocin } \\
(n=25)\end{array}$ & $P$ value & $\begin{array}{l}\text { Oxytocin } \\
(n=138)\end{array}$ & $\begin{array}{l}\text { Carbetocin } \\
(n=36)\end{array}$ & $P$ value & $\begin{array}{l}\text { Oxytocin } \\
(n=246)\end{array}$ & $\begin{array}{c}\text { Carbetocin } \\
(n=61)\end{array}$ & $P$ value \\
\hline Total blood loss $(\mathrm{mL}) \dagger$ & $451.4 \pm 269.6$ & $456.0 \pm 278.5$ & 0.94 & $529.1 \pm 951.4$ & $507.0 \pm 329.1$ & 0.82 & $492.6 \pm 722.2$ & $486.1 \pm 308.0$ & 0.92 \\
\hline Operating time (minutes) $\dagger$ & $43.1 \pm 18.3$ & $43.4 \pm 16.8$ & 0.93 & $43.8 \pm 26.3$ & $43.4 \pm 19.0$ & 0.92 & $43.5 \pm 23.2$ & $43.4 \pm 18.0$ & 0.97 \\
\hline Postpartum haemorrhage $\ddagger$ & $7(6.5 \%)$ & $1(4.0 \%)$ & 0.64 & $7(5.1 \%)$ & $2(5.6 \%)$ & 0.95 & $14(5.7 \%)$ & $3(4.9 \%)$ & 0.81 \\
\hline $\begin{array}{l}\text { Additional uterotonic } \\
\text { agents or procedures } \neq\end{array}$ & $5(4.6 \%)$ & $1(4.0 \%)$ & 0.89 & $6(4.3 \%)$ & $2(5.6 \%)$ & 0.79 & $11(4.5 \%)$ & $3(4.9 \%)$ & 0.88 \\
\hline Blood transfusion $\ddagger$ & $4(3.7 \%)$ & 0 & 0.33 & $6(4.3 \%)$ & $2(5.6 \%)$ & 0.79 & $10(4.1 \%)$ & $2(3.3 \%)$ & 0.77 \\
\hline Hysterectomy§ & 0 & 0 & - & $3(2.2 \%)$ & 0 & 1.00 & $3(1.2 \%)$ & 0 & 1.00 \\
\hline
\end{tabular}

\footnotetext{
* Data are shown as No. (\%) or mean \pm standard deviation, unless otherwise specified

+ Data calculated using Student's $t$ test

‡ Data calculated using Chi squared test

$\S$ Data calculated using Fisher's exact test
} 
TABLE 3. (cont'd)

(e) Fetal body weight $>4 \mathrm{~kg} *$

\begin{tabular}{|c|c|c|c|c|c|c|c|c|c|}
\hline & \multicolumn{3}{|c|}{ Elective Caesarean section } & \multicolumn{3}{|c|}{ Emergency Caesarean section } & \multicolumn{3}{|c|}{ Total } \\
\hline & $\begin{array}{l}\text { Oxytocin } \\
(n=44)\end{array}$ & $\begin{array}{l}\text { Carbetocin } \\
(n=8)\end{array}$ & $P$ value & $\begin{array}{l}\text { Oxytocin } \\
(n=69)\end{array}$ & $\begin{array}{c}\text { Carbetocin } \\
(n=13)\end{array}$ & $P$ value & $\begin{array}{c}\text { Oxytocin } \\
(n=113)\end{array}$ & $\begin{array}{l}\text { Carbetocin } \\
(n=21)\end{array}$ & $P$ value \\
\hline Total blood loss $(\mathrm{mL}) \dagger$ & $514.8 \pm 276.7$ & $418.8 \pm 116.8$ & 0.20 & $431.6 \pm 200.7$ & $480.8 \pm 235.0$ & 0.49 & $461.5 \pm 236.7$ & $457.1 \pm 209.3$ & 0.93 \\
\hline Operating time (minutes) $†$ & $40.4 \pm 13.6$ & $39.9 \pm 11.0$ & 0.91 & $41.8 \pm 13.0$ & $41.5 \pm 12.2$ & 0.93 & $41.2 \pm 13.1$ & $40.9 \pm 11.5$ & 0.90 \\
\hline Postpartum haemorrhage $\ddagger$ & $5(11.4 \%)$ & 0 & 0.32 & $1(1.4 \%)$ & $1(7.7 \%)$ & 0.19 & $6(5.3 \%)$ & $1(4.8 \%)$ & 0.92 \\
\hline $\begin{array}{l}\text { Additional uterotonic } \\
\text { agents or procedures } \ddagger\end{array}$ & $3(6.8 \%)$ & 0 & 0.45 & $2(2.9 \%)$ & $2(15.4 \%)$ & 0.06 & $5(4.4 \%)$ & $2(9.5 \%)$ & 0.34 \\
\hline Blood transfusion $\ddagger$ & $2(4.5 \%)$ & 0 & 0.54 & $1(1.4 \%)$ & $1(7.7 \%)$ & 0.19 & $3(2.7 \%)$ & $1(4.8 \%)$ & 0.60 \\
\hline Hysterectomy§ & 0 & 0 & - & 0 & 0 & - & 0 & 0 & - \\
\hline
\end{tabular}

(f) Polyhydramnios

\begin{tabular}{|c|c|c|c|c|c|c|c|c|c|}
\hline & \multicolumn{3}{|c|}{ Elective Caesarean section } & \multicolumn{3}{|c|}{ Emergency Caesarean section } & \multicolumn{3}{|c|}{ Total } \\
\hline & $\begin{array}{c}\text { Oxytocin } \\
(n=96)\end{array}$ & $\begin{array}{c}\text { Carbetocin } \\
(n=55)\end{array}$ & $P$ value & $\begin{array}{c}\text { Oxytocin } \\
(n=83)\end{array}$ & $\begin{array}{c}\text { Carbetocin } \\
(n=71)\end{array}$ & $P$ value & $\begin{array}{l}\text { Oxytocin } \\
(n=179)\end{array}$ & $\begin{array}{c}\text { Carbetocin } \\
(n=126)\end{array}$ & $P$ value \\
\hline Total blood loss (mL)† & $493.2 \pm 269.4$ & $531.8 \pm 782.8$ & 0.73 & $447.1 \pm 272.7$ & $505.6 \pm 308.2$ & 0.21 & $471.8 \pm 271.1$ & $516.4 \pm 560.2$ & 0.41 \\
\hline Operating time (minutes) $\dagger$ & $38.3 \pm 13.1$ & $45.2 \pm 35.7$ & 0.17 & $39.4 \pm 18.5$ & $38.3 \pm 10.0$ & 0.64 & $38.8 \pm 15.8$ & $41.5 \pm 24.8$ & 0.29 \\
\hline Postpartum haemorrhage $\neq$ & $10(10.4 \%)$ & $3(5.5 \%)$ & 0.30 & $4(4.8 \%)$ & $5(7.0 \%)$ & 0.57 & $14(7.8 \%)$ & $8(6.3 \%)$ & 0.60 \\
\hline $\begin{array}{l}\text { Additional uterotonic } \\
\text { agents or procedures } \neq\end{array}$ & $6(6.3 \%)$ & $2(3.6 \%)$ & 0.49 & $2(2.4 \%)$ & $5(7.0 \%)$ & 0.18 & $8(4.5 \%)$ & $7(5.6 \%)$ & 0.69 \\
\hline Blood transfusion $\ddagger$ & $6(6.3 \%)$ & $5(9.1 \%)$ & 0.52 & $1(1.2 \%)$ & $4(5.6 \%)$ & 0.13 & $7(3.9 \%)$ & $9(7.1 \%)$ & 0.14 \\
\hline Hysterectomy§ & 0 & 0 & - & 0 & 0 & - & 0 & 0 & - \\
\hline
\end{tabular}

TABLE 4. Linear regression and multiple logistic regression analysis regarding associations between carbetocin treatment and obstetric outcomes*

\begin{tabular}{|c|c|c|c|c|c|c|c|}
\hline & $\begin{array}{l}\text { All high-risk } \\
\text { cases }\end{array}$ & Fibroids & Macrosomia & Polyhydramnios & $\begin{array}{c}\text { Placenta praevia } \\
\text { (minor type) }\end{array}$ & $\begin{array}{l}\text { Placenta praevia } \\
\text { (major type) }\end{array}$ & $\begin{array}{l}\text { Twins } \\
\text { pregnancy }\end{array}$ \\
\hline Total blood loss (mL) & $\begin{array}{c}-14.19 \\
(-93.11 \text { to } 64.74)\end{array}$ & $\begin{array}{c}-62.64 \\
(-254.99 \text { to } 129.71)\end{array}$ & $\begin{array}{c}-2.98 \\
(-112.22 \text { to }-106.28)\end{array}$ & $\begin{array}{c}18.54 \\
(-76.68 \text { to } 113.77)\end{array}$ & $\begin{array}{c}-91.08 \\
(-230.20 \text { to } 48.05)\end{array}$ & $\begin{array}{c}-371.23 \\
(-905.90 \text { to } 163.47)\end{array}$ & $\begin{array}{c}-142.90 \\
(-249.50 \text { to } 8.69)\end{array}$ \\
\hline Operating time $(\mathrm{min}) \dagger$ & $\begin{array}{c}1.06 \\
(-1.44 \text { to } 3.56)\end{array}$ & $\begin{array}{c}-1.39 \\
(-7.73 \text { to } 4.95)\end{array}$ & $\begin{array}{c}-0.49 \\
(-6.29 \text { to } 5.31)\end{array}$ & $\begin{array}{c}2.32 \\
(-2.35 \text { to } 7.00)\end{array}$ & $\begin{array}{c}2.78 \\
(-2.35 \text { to } 7.91)\end{array}$ & $\begin{array}{c}-2.36 \\
(-16.87 \text { to } 12.15)\end{array}$ & $\begin{array}{c}-0.47( \\
-5.09 \text { to } 4.16)\end{array}$ \\
\hline $\begin{array}{l}\text { Postpartum } \\
\text { haemorrhage } \neq\end{array}$ & $\begin{array}{c}0.87 \\
(0.56-1.35)\end{array}$ & $\begin{array}{c}0.81 \\
(0.20-3.24)\end{array}$ & $\begin{array}{c}0.85 \\
(0.08-8.74)\end{array}$ & $\begin{array}{c}0.61 \\
(0.24-1.56)\end{array}$ & $\begin{array}{c}0.92 \\
(0.26-3.30)\end{array}$ & $\begin{array}{c}0.25 \\
(0.08-0.78)\end{array}$ & $\begin{array}{c}0.48 \\
(0.24-0.94)\end{array}$ \\
\hline $\begin{array}{l}\text { Additional } \\
\text { uterotonic agents or } \\
\text { procedures } \ddagger\end{array}$ & $\begin{array}{c}0.78 \\
(0.47-1.28)\end{array}$ & $\begin{array}{c}0.99 \\
(0.23-4.18)\end{array}$ & $\begin{array}{c}2.08 \\
(0.34-12.57)\end{array}$ & $\begin{array}{c}1.03 \\
(0.35-3.00)\end{array}$ & $\begin{array}{c}0.63 \\
(0.13-2.96)\end{array}$ & $\begin{array}{c}0.23 \\
(0.06-0.80)\end{array}$ & $\begin{array}{c}0.39 \\
(0.18-0.88)\end{array}$ \\
\hline Blood transfusion $\ddagger$ & $\begin{array}{c}1.48 \\
(0.93-2.35)\end{array}$ & $\begin{array}{c}0.50 \\
(0.09-2.68)\end{array}$ & $\begin{array}{c}1.27 \\
(0.08-20.37)\end{array}$ & $\begin{array}{c}1.55 \\
(0.54-4.43)\end{array}$ & $\begin{array}{c}0.75 \\
(0.17-3.33)\end{array}$ & $\begin{array}{c}1.17 \\
(0.41-3.29)\end{array}$ & $\begin{array}{c}1.37 \\
(0.71-2.61)\end{array}$ \\
\hline
\end{tabular}

* Sample for hysterectomy was too small for logistic regression

† Data calculated by linear regression, presented as unstandardised regression coefficient B (95\% confidence intervals)

‡ Data calculated by multiple logistic regression, presented as adjusted odds ratio ( $95 \%$ confidence intervals)

When we analysed individual risk factors for multiple pregnancies. Our results are consistent postpartum haemorrhage, we found significant with the findings of a previous study in which lower reductions in the use of additional uterotonics haemoglobin and haematocrit differences were or procedures and in the rate of postpartum found in women who received carbetocin treatment haemorrhage in women with major placenta praevia during Caesarean section due to multiple gestation and in women with multiple pregnancies. We also or placenta praevia, compared with women who observed a significant reduction in total blood loss received oxytocin treatment. ${ }^{15}$ Carbetocin can in the emergency Caesarean section group for both induce strong contraction of an overdistended uterus women with major placenta praevia and women with associated with twin pregnancies. ${ }^{16}$ However, a study 
in 2013 did not show beneficial effects of carbetocin administration. ${ }^{17}$ The sample size was small in that study and its design comprised a retrospective before-and-after analysis. Additionally, we presume that the beneficial effect of carbetocin in reduction of bleeding in women with placenta praevia might be related to its effectiveness in stimulating the retroplacental myometrium. ${ }^{18}$ Carbetocin can shorten the third stage, prevent and treat retained placenta at term, and prevent and treat second trimester abortion. ${ }^{19}$ However, our results were inconsistent with the findings of a previous study, in which the additional effects of carbetocin were presumed to be trivial because of thinning in the lower uterine myometrium, thereby reducing the immunoreactivity of oxytocin receptors relative to the upper part of the uterus. ${ }^{20}$

We noted that the significant differences in outcomes between treatments were mainly due to the contributions of the emergency Caesarean section group. Previous studies have largely demonstrated beneficial effects from carbetocin treatment in women undergoing elective Caesarean section, but not in women undergoing intrapartum Caesarean section. ${ }^{15,21}$ A possible explanation might be that emergency Caesarean sections were performed before the date of scheduled Caesarean section date; hence, the gestational age and fetal weight were typically lower at the time of the operation.

The greatest strength of this study was that it included a relatively large sample size, which comprised 1236 patients in one hospital with standard protocols. There were a few limitations in this study. First, it used single-centre, retrospective design. Second, complete blood count data were not routinely collected before delivery during the study period, so these data could not be compared among subgroups. Third, the implementation of carbetocin began in April 2017. Since this implementation, most patients with the risk factors considered in this study were administered carbetocin instead of oxytocin infusion, in accordance with our department protocol. This led to a comparison between different time frames, during which there were changes in medical personnel and training. Finally, judgement regarding the use of additional uterotonic agents and transfusion may have differed among attending physicians.

In clinical practice, the use of carbetocin has been acknowledged in guidelines from the Society of Obstetricians and Gynaecologists of Canada $^{10}$ and the Royal College of Obstetricians and Gynaecologists. ${ }^{9}$ We recommend the use of carbetocin during Caesarean section for women with multiple pregnancies and major placenta praevia, based on the beneficial effects demonstrated in the present study and in prior studies. ${ }^{15}$ In women with other risk factors for postpartum haemorrhage, we recommend the use of oxytocin infusion, instead of carbetocin. ${ }^{15}$ Larger studies or prospective trials are needed to investigate the effectiveness of carbetocin during Caesarean section for different indications and in women with risk factors for postpartum haemorrhage; such studies are also needed to establish the cost-effectiveness of this relatively new drug.

In conclusion, we found that carbetocin and oxytocin infusion had differential effects on the requirement for additional uterotonics or procedures in women who underwent Caesarean section for different indications. In particular, compared with oxytocin infusion, carbetocin was associated with a reduction in the requirement for additional uterotonics or procedures for women with multiple pregnancies and women with major placenta praevia.

\section{Author contributions}

Concept or design: All authors.

Acquisition of data: KY Tse, FNY Yu.

Analysis or interpretation of data: KY Tse, FNY Yu.

Drafting of the manuscript: KY Tse, KY Leung.

Critical revision of the manuscript for important intellectual content: KY Tse, KY Leung.

All authors had full access to the data, contributed to the study, approved the final version for publication, and take responsibility for its accuracy and integrity.

\section{Conflicts of interest}

As an editor of the journal, KY Leung was not involved in the peer review process. Other authors have no conflicts of interests to disclose.

\section{Funding/support}

This study did not receive any specific grant from funding agency in the public or commercial sectors.

\section{Ethics approval}

This study was approved by the Hospital Authority Kowloon Central/Kowloon East Research Ethics Committee (Ref KC/ KE-20-0073/ER-1).

\section{References}

1. Say L, Chou D, Gemmill A, et al. Global causes of maternal death: a WHO systematic analysis. Lancet Glob Health 2014;2:e323-33.

2. Anderson JM, Etches D. Prevention and management of postpartum haemorrhage. Am Fam Physician 2007;75:87582.

3. Su LL, Chong YS, Samuel M. Carbetocin for preventing postpartum haemorrhage. Cochrane Database Syst Rev 2012;(4):CD005457.

4. Fahmy NG, Yousef HM, Zaki HV. Comparative study between effect of carbetocin and oxytocin on isofluraneinduced uterine hypotonia in twin pregnancy patients undergoing cesarean section. Egypt J Anaesth 2016;32:11721. 
5. Hunter DJ, Schulz P, Wassenaar W. Effect of carbetocin, a long-acting oxytocin analog on the postpartum uterus. Clin Pharmacol Ther 1992;52:60-7.

6. Voon HY, Suharjono HN, Shafie AA, Bujang MA. Carbetocin versus oxytocin for the prevention of postpartum hemorrhage: a meta-analysis of randomized controlled trials in cesarean deliveries. Taiwan J Obstet Gynecol 2018;57:332-9.

7. Kalafat E, Gokce A, O'Brien P, et al. Efficacy of carbetocin in the prevention of postpartum hemorrhage: a systematic review and Bayesian meta-analysis of randomized trials. J Matern Fetal Neonatal Med 2019 Sep 19. Epub ahead of print.

8. Onwochei DN, Van Ross J, Singh PM, Salter A, Monks DT. Carbetocin reduces the need for additional uterotonics in elective Caesarean delivery: a systematic review, metaanalysis and trial sequential analysis of randomised controlled trials. Int J Obstet Anesth 2019;40:14-23.

9. Mavrides E, Allard S, Chandraharan E, et al. Prevention and management of postpartum haemorrhage. Green-top Guideline No. 52. BJOG 2016;124:e106-49.

10. Leduc D, Senikas V, Lalonde AB. No. 235-Active management of the third stage of labour: prevention and treatment of postpartum hemorrhage. J Obstet Gynaecol Can 2018;40:e841-55.

11. El Behery MM, El Sayed GA, El Hameed AA, Soliman BS, Abdelsalm WA, Bahaa A. Carbetocin versus oxytocin for prevention of postpartum hemorrhage in obese nulliparous women undergoing emergency cesarean delivery. J Matern Fetal Neonatal Med 2016;29:1257-60.

12. Chen CY, Su YN, Lin TH, et al. Carbetocin in prevention of postpartum hemorrhage: experience in a tertiary medical center of Taiwan. Taiwan J Obstet Gynecol 2016;55:804-9.

13. Mohamed Maged A, Ragab AS, Elnassery N, Ai Mostafa W, Dahab S, Kotb A. Carbetocin versus syntometrine for prevention of postpartum hemorrhage after cesarean section. J Matern Fetal Neonatal Med 2017;30:962-6.

14. Borruto F, Treisser A, Comparetto C. Utilization of carbetocin for prevention of postpartum hemorrhage after cesarean section: a randomized clinical trial. Arch Gynecol Obstet 2009;280:707-12.

15. Chen YT, Chen SF, Hsieh TT, Lo LM, Hung TH. A comparison of the efficacy of carbetocin and oxytocin on hemorrhage-related changes in women with cesarean deliveries for different indications. Taiwan J Obstet Gynecol 2018;57:677-82.

16. Seow KM, Chen KH, Wang PH, Lin YH, Kwang JL. Carbetocin versus oxytocin for prevention of postpartum hemorrhage in infertile women with twin pregnancy undergoing elective cesarean delivery. Taiwan J Obstet Gynecol 2017;56:273-5.

17. Demetz J, Clougueur E, D'Haveloose A, Staelen P, Ducloy AS, Subtil D. Systematic use of carbetocin during cesarean delivery of multiple pregnancies: a before-and-after study. Arch Gynecol Obstet 2013;287:875-80.

18. Abbas AM. Different routes and forms of uterotonics for treatment of retained placenta: methodological issues. J Matern Fetal Neonatal Med 2017;30:2179-84.

19. Elfayomy AK. Carbetocin versus intra-umbilical oxytocin in the management of retained placenta: a randomized clinical study. J Obstet Gynaecol Res 2015;41:1207-13.

20. Kato S, Tanabe A, Kanki K, et al. Local injection of vasopressin reduces the blood loss during cesarean section in placenta previa. J Obstet Gynaecol Res 2014;40:1249-56.

21. Elbohoty AE, Mohammed WE, Sweed M, Bahaa Eldin AM, Nabhan A, Abd-El-Maeboud KH. Randomized controlled trial comparing carbetocin, misoprostol, and oxytocin for the prevention of postpartum hemorrhage following an elective cesarean delivery. Int J Gynaecol Obstet 2016;34:324-8. 